\title{
APPLICATION OF HILL FUNCTIONS TO CIRCULAR PLATE PROBLEMS*
}

\author{
$\mathrm{BY}$ \\ ROBERT KAO \\ The Catholic University of America, Washington, D.C.
}

\begin{abstract}
A type of finite element-hill functions-is applied to solve circular plate problems in conjunction with the method of Lagrange multipliers which is used to treat various constraint conditions. Results obtained compare very nicely with the exact solutions.
\end{abstract}

Introduction. Finite-element methods have been widely applied to obtain numerical solutions in both engineering $[1,2]$ and applied mathematics $[3,4,5]$. The popularity of the methods may be partially due to their ability to deal with more complex problems and to provide accurate numerical results. However, it should be noted that there are different kinds of finite-element methods and the use of hill functions as finite elements which are developed in applied mathematics $[3,4,5,6]$ is one of them.

In [6], hill functions are utilized to solve one-dimensional string-beam problems in which the so-called "method of artificial parameters" is used for handling various boundary conditions. In the present paper, these same functions are employed to solve axisymmetric (polar coordinate) circular plate problems; the Lagrange multiplier method is used here for the treatment of constraint situations. The striking difference between these two types of applications is in the evaluation of matrix elements of the system equations; for string-beam problems, all the terms in these elements turn out to be simply multiples of hill function coefficients [6], but for circular plate problems, they are not so straight-forward and require, for evaluation, methods of numerical integration.

Rayleigh-Ritz method. For the sake of completeness and use in the following sections, key equations involved in the Rayleigh-Ritz method are presented in this section.

Suppose there exists a sequence $y_{1}, y_{2}, \cdots$ of admissible functions in the variational problem such that

$$
\lim _{n \rightarrow \infty} F\left(y_{n}\right)=d,
$$

where $d$ is the lower bound of the functional $F(y)$. The Rayleigh-Ritz method is a recipe for the construction of such a sequence by choosing an arbitrary system of coordinate functions, $\omega_{1}, \omega_{2}, \cdots$, with the property that any linear combination

$$
y_{n}=c_{1} \omega_{1}+c_{2} \omega_{2}+\cdots+c_{n} \omega_{n}
$$

is admissible in the variational problem, and that the solution function $y$ and its relevant

${ }^{*}$ Received October 26,1973 . The research reported here was supported by the Office of Naval Research (N00014-67-A-0377-0011). 
derivatives may be approximated with any degree of accuracy by Eq. (2) and its corresponding derivatives, respectively.

If the problem under consideration, $F(y)$, is a quadratic functional, then the values $c_{i}$ can be determined by $n$ linear simultaneous equations

$$
\frac{\partial F\left(y_{n}\right)}{\partial c_{i}}=0, \quad i=1,2, \cdots, n .
$$

Problem formulation and system equations. For simplicity, the axisymmetric case of circular plate problems will be considered herein (Fig. 1), in which the load is axisymmetrically distributed but allowed to vary along the radial direction $r$. The governing differential equation is given [7] as

$$
\frac{1}{r} \frac{d}{d r}\left\{r \frac{d}{d r}\left[\frac{1}{r} \frac{d}{d r}\left(r \frac{d y}{d r}\right)\right]\right\}=\frac{q(r)}{D}
$$

where $y$ is normal deflection of the plate, $q$ is the loading intensity and is a function of $r$, and

$$
D=\frac{E t^{3}}{12\left(1-\nu^{2}\right)}
$$

in which $E$ is Young's modulus, $t$ is the thickness of the plate and $\nu$ is Poisson's ratio.

For generality, the constraint conditions associated with Fig. 1 may be expressed by

$$
\begin{aligned}
& \Phi_{1}\left(y_{0}, y_{0}{ }^{\prime}, y_{0}{ }^{\prime \prime}\right)=0 \\
& \Phi_{2}\left(y_{a}, y_{a}{ }^{\prime}, y_{a}{ }^{\prime \prime}\right)=0 \\
& \Phi_{3}\left(y_{a}, y_{a}{ }^{\prime}, y_{a}{ }^{\prime \prime}\right)=0
\end{aligned}
$$

where $y_{0}=y(0), y_{a}=y(a)$, and ()$^{\prime}=(d / d r)()$.

The energy expression corresponding to Eq. (4) is also given [7] as follows:

$$
F_{1}=\pi \int_{0}^{a}\left\{D\left[\left(y^{\prime \prime}\right)^{2}+2 \nu \frac{y^{\prime}}{r} y^{\prime \prime}+\left(\frac{y^{\prime}}{r}\right)^{2}\right]-2 y q\right\} r d r .
$$

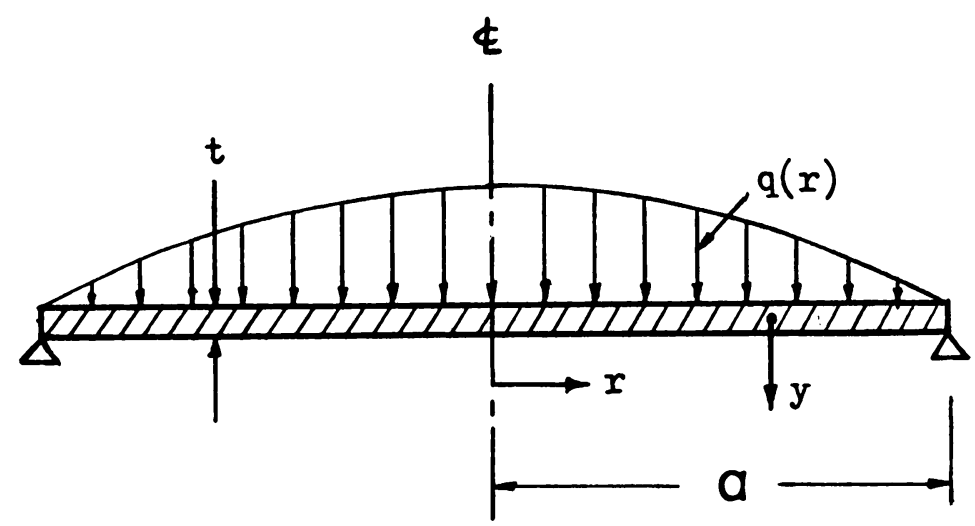

Fig. 1. Circular plate under axisymmetric load. 
Substituting Eq. (2) into this expression yields a functional of $n$ undetermined constants which may be put into a form of

$$
F_{1}=\frac{1}{2}\lfloor c\rfloor[K]\{c\}-\lfloor c\rfloor\{q\}
$$

where $\lfloor c\rfloor=\left\lfloor c_{1}, c_{2}, \cdots, c_{n}\right\rfloor,[K]=$ stiffness matrix of system of equations (4), and $\{q\}=$ column matrix related to applied loading.

Before the Rayleigh-Ritz method can be applied to this functional, account must be taken of constraint conditions, Eqs. (6), which may be written in matrix notation as

$$
\{\Phi\}=0
$$

where $\lfloor\Phi\rfloor=\left\lfloor\Phi_{1}, \Phi_{2}, \Phi_{3}\right\rfloor$. Substitution of Eq. (2) into Eq. (9) yields

$$
\{\Phi\}=[\Gamma]\{c\}=0
$$

In the Rayleigh-Ritz method, Eqs. (3) (which have $n$ equations) are not all independent since they are related to each other by the constraint equations (6). Consequently, Eqs. (6) can be viewed as three extra conditions on this problem. The procedure employed here to eliminate these extra conditions is the classical method of Lagrange multipliers $[8,9]$. We should here define a multiplier $\lambda_{i}$ for each of constraint equations. The product of the vector of Lagrange multipliers, $\lfloor\lambda\rfloor=\left\lfloor\lambda_{1}, \lambda_{2}, \lambda_{3}\right\rfloor$, and constraint equations (10) is added to the original functional (Eq. (8)), i.e. (with $L \lambda\rfloor[\Gamma]\{c\}=$ $\left.\lfloor c\rfloor[\Gamma]^{T}\{\lambda\}\right)$

$$
F=\frac{1}{2}\lfloor c\rfloor[K]\{c\}-\lfloor c\rfloor\{q\}+\lfloor c\rfloor[\Gamma]^{T}\{\lambda\} .
$$

Application of Eqs. (3) with variation of all parameters in $\{c\}$ and $\{\lambda\}$ gives

$$
\begin{aligned}
& \begin{array}{c}
\uparrow \\
n \\
\frac{\downarrow}{\uparrow} \\
3 \\
\downarrow
\end{array}\left[\begin{array}{c:c}
K & \Gamma^{T} \\
\hdashline \Gamma & 0
\end{array}\right]\left\{\begin{array}{c}
c \\
- \\
\lambda
\end{array}\right\}=\left\{\begin{array}{c}
q \\
\hdashline \\
0
\end{array}\right\} . \\
& |\leftarrow n \rightarrow| \leftarrow 3 \rightarrow \mid
\end{aligned}
$$

Eq. (12a) is a symmetric matrix and can be solved for $\{c\}$ and $\{\lambda\}$ provided that coordinate functions in Eq. (2) have been properly selected. In this paper, a special type of coordinate functions-hill functions-is used for all computations, a brief description of which will be given in the next section.

A different arrangement of constraint conditions in (11a) may result in a symmetric, banded-width matrix. This new arrangement is

$$
F=\lfloor c\rfloor\left[\Gamma_{1}\right]\left\{\bar{\lambda}_{1}\right\}+\frac{1}{2}\lfloor c\rfloor[K]\{c\}-\lfloor c\rfloor\{q\}+\lfloor c\rfloor\left[\Gamma_{2}\right]^{T}\left\{\bar{\lambda}_{2}\right\}
$$

where $\left\lfloor\bar{\lambda}_{1}\right\rfloor=\left\lfloor\lambda_{1}\right\rfloor=\lambda_{1}$ and $\left\lfloor\bar{\lambda}_{2}\right\rfloor=\left\lfloor\lambda_{2}, \lambda_{3}\right\rfloor$. 
A set of equations similar to Eqs. (12a) may be readily obtained as follows:

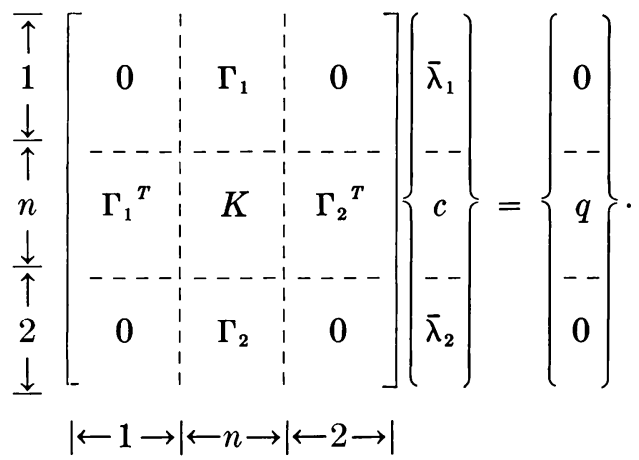

To obtain a solution to Eqs. (12b) by using hill functions as coordinate functions, advantage can be taken of the properties of symmetric, banded-width matrix so that less computing time than for solving Eqs. (12a) can generally be expected.

Coordinate functions-hill functions. Some finite element models called "hill functions" have recently been developed $[3,4,5,6]$. These models, which can hardly satisfy given boundary conditions without special considerations but are quite economic when used in numerical computation, are utilized as coordinate functions in this paper.

The construction of hill functions has been described in fair detail in $[3,6]$; here an outline only of the equations is given:

$$
\begin{gathered}
{ }^{n} \phi(x)=\sum_{i=1}^{n}{ }^{n} \phi_{j}(\xi), \quad-n / 2 \leq x \leq n / 2 \\
{ }^{n} \phi_{i}(\xi)=\sum_{i=1}^{n}{ }^{n} \alpha_{i, i} P_{i}(\xi), \quad-1 / 2 \leq \xi \leq 1 / 2
\end{gathered}
$$

where ${ }^{n} \phi(x)$ denotes the hill function of order $n ; x$ and $\xi$ represent the global and local coordinate systems, respectively.

The graphical representation of Eqs. (13) are sketched for $n=4,5$ in Fig. 2. From this figure we note that the entire domain of ${ }^{n} \phi(x),-n / 2 \leq x \leq n / 2$, is divided into $n$ even intervals, and in each interval a local coordinate system $(-1 / 2 \leq \xi \leq 1 / 2)$ is set up having the origin at the interval center. Thus, ${ }^{n} \phi(x)$ can be taken as a sum of $n$ portions, Eq. (13a), and each portion of this function is represented in the local coordinate system by a Fourier series expansion in terms of Legendre polynomials $P_{i}(\xi)$ (with $\left.P_{1}(\xi)=1\right)$, Eq. (13b) $\left({ }^{n} \alpha_{i, i}\right.$ in this equation are coefficient constants).

Derivative expressions of hill functions may also be given as follows:

$$
\begin{aligned}
& { }^{n} \phi_{1}{ }^{(k)}(\xi)={ }^{n-1} \phi_{1}{ }^{(k-1)}(\xi), \quad{ }^{n} \phi_{n}{ }^{(k)}(\xi)=-{ }^{n-1} \phi_{n-1}{ }^{(k-1)}(\xi), \\
& { }^{n} \phi_{i}{ }^{(k)}(\xi)={ }^{n-1} \phi_{i}{ }^{(k-1)}(\xi)-{ }^{n-1} \phi_{i-1}{ }^{(k-1)}(\xi) \text { for } j=2, \cdots, n-1,
\end{aligned}
$$

or

$$
\begin{aligned}
{ }^{n} \phi_{i}{ }^{(k)}(\xi)=\sum_{i=0}^{k}(-1)^{i}\left(\begin{array}{c}
k \\
i
\end{array}\right){ }^{n-k} \phi_{i-i}(\xi) & \\
& (k=1, \cdots, n-2 ; j=1, \cdots, n ; 1 \leq j-i \leq n-k),
\end{aligned}
$$




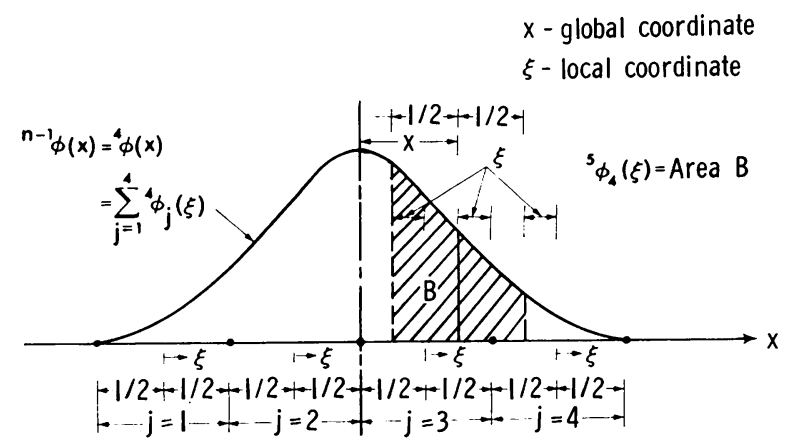

(a)

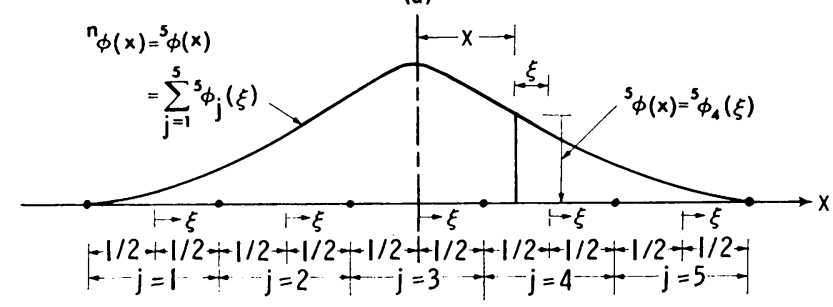

(b)

FIg. 2. Construction of hill functions, Eqs. (13).

and

$$
{ }^{n} \phi_{j}{ }^{(k)}(\xi)=\sum_{i=1}^{n-k}{ }^{n} b_{i, i}{ }^{(k)} P_{i}(\xi), \quad j=1,2, \cdots, n
$$

in which the superscript $(k)$ denotes the order of derivatives. The coefficients ${ }^{n} b_{i, i}{ }^{(k)}$ can be obtained in terms of ${ }^{n-k} a_{i, j}$ through Eq. (14a).

The numerical values of ${ }^{n} a_{i, j}$ with $n$ ranging from 1 to 4 are given in Table 1 ; the hill functions corresponding to these values are plotted in Fig. 3. Incidentally, a computer program has been developed which computes ${ }^{n} a_{i, 2}$ and ${ }^{n} b_{i, j}{ }^{(k)}$ up to any order desired.

Expression of stiffness matrix [K]. A special type of coordinate functions-hill functions, summarized in the previous section-will be utilized herein for all calculations.

TABLE 1.*

${ }^{n} a_{i, j}$ in Eqs. (13) $(n=1, \cdots, 4)$.

\begin{tabular}{ccllcc}
\hline$n$ & $j$ & $i=1$ & $i=2$ & $i=3$ & $i=4$ \\
\hline 1 & 1 & 1 & & & \\
2 & 1 & 0.5 & 0.5 & & \\
3 & 1 & 0.1667 & 0.25 & 0.08333 & \\
& 2 & 0.6667 & 0 & -0.1666 & 0.008333 \\
4 & 1 & 0.04167 & 0.075 & 0.04167 & -0.025 \\
\hline
\end{tabular}

* Because of the symmetry of hill functions about $x=0$, only symmetric parts of coefficients are given; for more accurate values, a double precision version may be used in the computer code. 


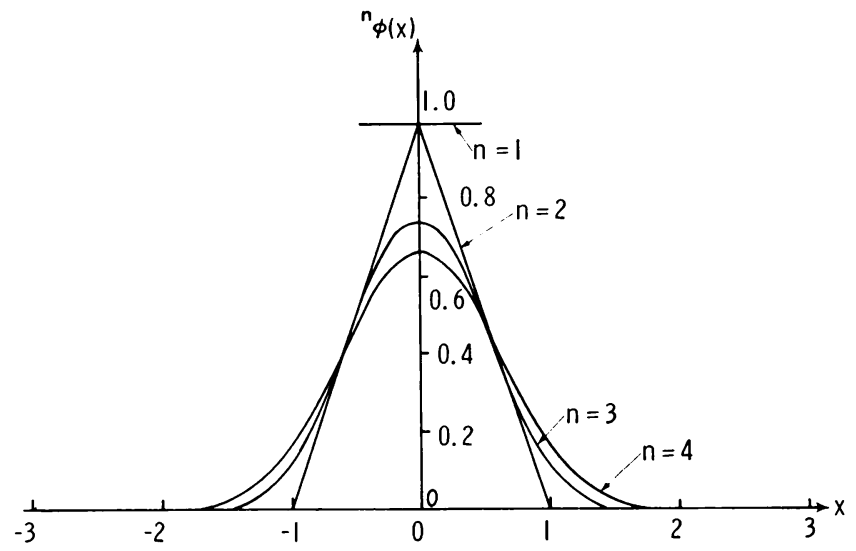

FIg. 3. Profiles of hill functions ${ }^{n} \phi(x)$, Eqs. (13), with $1 \leq n \leq 4$.

Before going to solve Eqs. (12), we shall here give an expression for the stiffness matrix $[K]$ of Eqs. (12); the derivations of $[\Gamma]$ and $\{q\}$ may be quite straightforward and will depend on constraint conditions and loading situations, respectively, in each individual case.

In order to suit the dimension requirements specified in the hill function description, the following dimensionless quantities are introduced:

$$
r=h \bar{r}, \quad y=h \bar{y}, \quad h=a_{\prime}^{\prime} m
$$

where $m$ is the total number of intervals into which the entire domain $(0 \leq r \leq a)$ is divided.

According to Eq. (2), the deflection function $\bar{y}$ is now expressed in terms of hill functions by

$$
\bar{y}_{h}(\bar{r})=\sum_{i=1}^{m+n-1} c_{i}^{n} \phi(\bar{r}-j) .
$$

A graphical representation of Eq. (16) is sketched in Fig. 4 for $n=4$ and $m=5$.

By use of Eq. (16), a generic term of $[K]$ may be given as

$$
\begin{aligned}
K_{i i}= & 2 \pi D\left\{\left[\bar{r} \phi^{\prime \prime}(\bar{r}-i), \phi^{\prime \prime}(\bar{r}-j)\right]\right. \\
& +\nu\left[\phi^{\prime}(\bar{r}-i), \phi^{\prime \prime}(\bar{r}-j)\right]+\nu\left[\phi^{\prime \prime}(\bar{r}-i), \phi^{\prime}(\bar{r}-j)\right] \\
& \left.+\left[\frac{1}{\bar{r}} \phi^{\prime}(\bar{r}-i), \phi^{\prime}(\bar{r}-j)\right]\right\}
\end{aligned}
$$

where

$$
\begin{aligned}
{\left[\bar{r} \phi^{\prime \prime}(\bar{r}-i), \phi^{\prime \prime}(\bar{r}-j)\right] } & =\int_{0}^{m}{ }^{n} \phi^{\prime \prime}(\bar{r}-i)^{n} \phi^{\prime \prime}(\bar{r}-j) \bar{r} d \bar{r} \\
{\left[\phi^{\prime}(\bar{r}-i), \phi^{\prime \prime}(\bar{r}-j)\right] } & =\int_{0}^{m}{ }^{n} \phi^{\prime}(\bar{r}-i)^{n} \phi^{\prime \prime}(\bar{r}-j) d \bar{r} \\
{\left[\phi^{\prime \prime}(\bar{r}-i), \phi^{\prime}(\bar{r}-j)\right] } & =\int_{0}^{m}{ }^{n} \phi^{\prime \prime}(\bar{r}-i)^{n} \phi^{\prime}(\bar{r}-j) d \bar{r}
\end{aligned}
$$




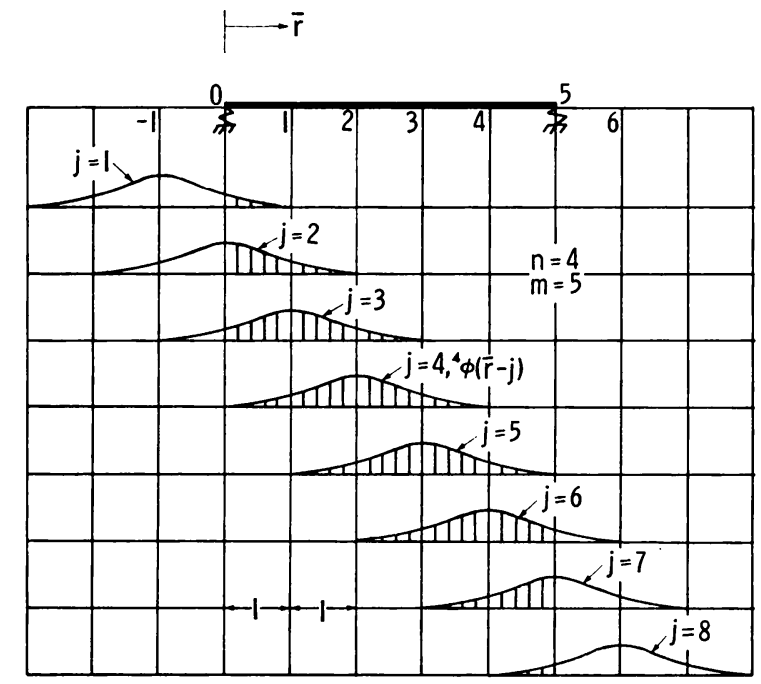

FIG. 4. Graphical representation of Eq. (16) with $n=4, m=5$.

$$
\left[\frac{1}{\bar{r}} \phi^{\prime}(\bar{r}-i), \phi^{\prime}(\bar{r}-j)\right]=\int_{0}^{m}{ }^{n} \phi^{\prime}(\bar{r}-i)^{n} \phi^{\prime}(\bar{r}-j) \frac{1}{\bar{r}} d \bar{r} .
$$

After substituting Eqs. (13) and its corresponding derivative expressions, Eqs. (14), into Eqs. (18), evaluation of these quantities can be obtained without any major difficulty. However, it should be realized that evaluations of Eqs. (18b, c) are performed interval by interval, utilizing the orthogonality of the Legendre polynomials in $(-1 / 2,1 / 2)$, i.e.,

$$
\begin{aligned}
\int_{-1 / 2}^{1 / 2} P_{i}(\xi) P_{i}(\xi) d \xi & =1 /(2 i-1), & & i=j ; \\
& =0, & & i \neq j .
\end{aligned}
$$

And these evaluations turn out to be simply some kind of multiplication of coefficients of hill function and their derivative expressions, ${ }^{n} a_{i, j}$ and ${ }^{n} b_{i, j}{ }^{(k)}$, a detailed example of which can be found in Ref. [6]. On the other hand, the integrals (18a, d) do not enjoy such simplicity due to existence of additional arguments $\bar{r}$ and $1 / \bar{r}$ respectively, and must be obtained numerically as follows: Eqs. (18a, d) are first taken as a sum of integrals in each interval, and these integrals are obtained by further splitting each interval into a number of subintervals and then employing the usual numerical integration schemes.

Here it is important to note that there is a singularity problem in Eq. (18d) when $\bar{r}=0$. Eq. (18d) comes from the third term of Eq. (7), $\int_{0}^{a}\left(y^{\prime} / r\right) r d r$. A close examination of this term reveals that, due to axisymmetricity, $y^{\prime}=0$ at the plate center, and an application of L'Hospital's rule yields $y^{\prime} / r=y^{\prime \prime}$. Therefore, the value of Eq. (18d) should be zero when evaluation of this quantity is performed at the plate center $(r=0)$.

Numerical results. Two example problems are solved using Eqs. (12b) as system equations with matrix band-width of 13 as a hill function of order $n=6$ is used. In both calculations, 10 mesh spacings $(0 \leqq r \leqq a)$ are adopted. Results are presented for 


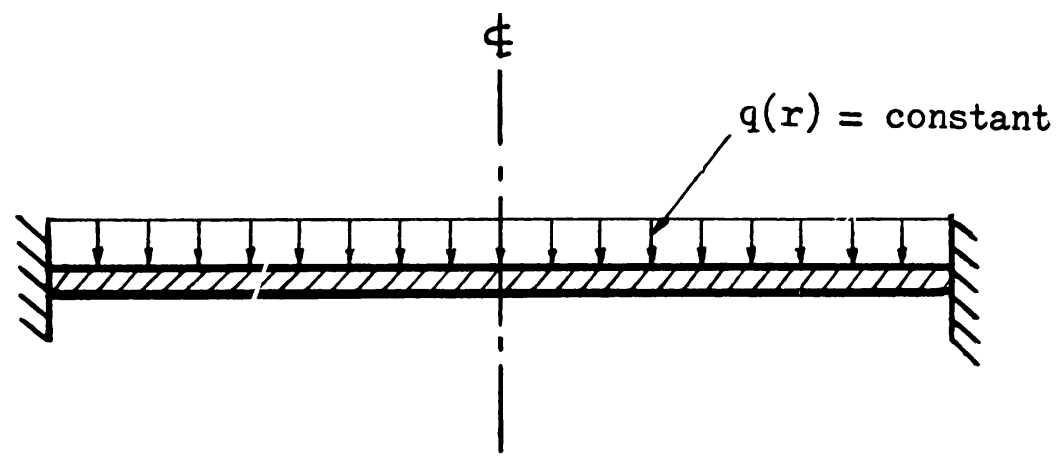

FIg. 5. Uniformly loaded solid circular plate with clamped edge.

the deflection and stresses $\left(\sigma_{r}\right.$ and $\left.\sigma_{\theta}\right)$ along the radial and circumferential directions; $\sigma_{r}$ and $\sigma_{\theta}$ are related to the defiection function by

$$
\begin{aligned}
\sigma_{r}=6 M M_{r} / t^{2}, & \sigma_{\theta}=6 M I_{\theta} / t^{2}, \\
M_{r}=-D\left(\frac{d^{2} y}{d r^{2}}+\frac{\nu}{r} \frac{d y}{d r}\right), & M_{\theta}=-D\left(\frac{1}{r} \frac{d y}{d r}+\nu \frac{d^{2} y}{d r^{2}}\right) .
\end{aligned}
$$

1. Uniformly loaded circular plate with clamped edge. This example problem is shown in Fig. 5 in which $q(r)$ is a constant function; constraint conditions are

$$
\Phi_{1}=y^{\prime}(0)=0, \quad \Phi_{2}=y(a)=0, \quad \Phi_{3}=y^{\prime}(a)=0 .
$$

The numerical results are displayed in Table 2. The computation, including construction of hill functions up to order $n=6$ and their corresponding derivatives, took 1.73 seconds of PDP-10 CPU time.

2. Circular plate with a central hole, uniformiy loaded along inner edge and simply supported along outer edge. In this case (Fig. 6), the total loading along the inner circle is $P=2 \pi b Q_{0}$, where $b$ is the radius of central hole, $Q_{0}$ is the intensity of uniformly

\section{TABLE 2}

Comparison of the present results and the exact solutions ([7], shown in parentheses) for a uniformly loaded clamped circular plate (Fig. 5) (the hill function of order $n=6$ and mesh spacings $m=10$ are used),

$$
\begin{array}{llllll}
\frac{r}{a}=0 & \frac{r}{a}=0.2 & \frac{r}{a}=0.4 & \frac{r}{a}=0.6 & \frac{r}{a}=0.8 & \frac{r}{a}=1
\end{array}
$$
(0.17062:)
0.157245
. 120393
0.022113
$0 \quad)$
0.170628
0.157233
0.120377
0.069888
(). 022098
0
(b) Stress along radial direction $\sigma_{r} t^{2} / q a^{2}$.
(0. 487500
0.438000
0.289500
0.042000
$-0.304500$
$-0.750000)$
0.505530
0.437587
o. 289040
0.040964
$-0.307187$
$-0.718076$

(c) Stress along circumferential direction $\sigma_{\theta} l^{2} / q a^{2}$.
$(0.487 .500$
0. 459000
0.373500
0. 231000
0. 031500
$-0.225000)$
0.505530
0.458961
0.373340
0. 2330637
$0.030: 59$
$-0.215423$ 


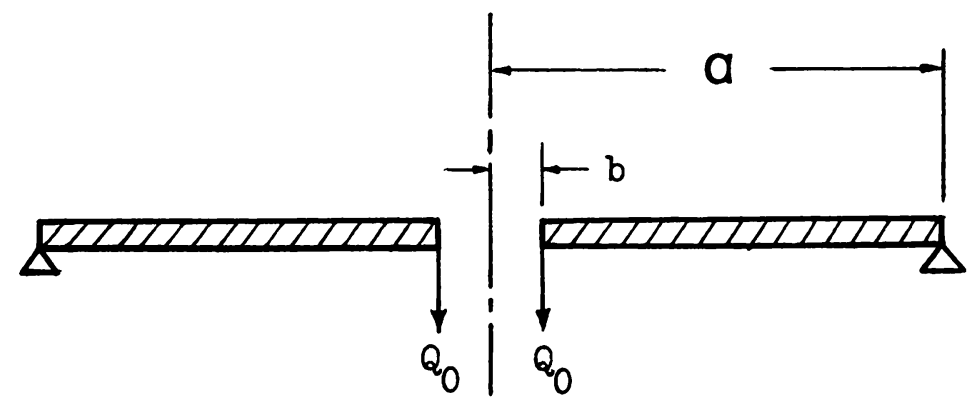

FIG. 6. Simply supported circular plate with a central hole.

distributed line load. The constraint conditions for this case are

$\Phi_{1}=y^{\prime \prime}(b)+\frac{\nu}{b} y^{\prime}(b)=0, \quad \Phi_{2}=y(a)=0, \quad \Phi_{3}=y^{\prime \prime}(a)+\frac{\nu}{a} y^{\prime}(a)=0$.

The numerical results are obtained for $b / a=1 / 10$ and presented in Table 3 . The computation for this problem took 1.42 seconds of PDP-10 CPU time.

Concluding remarks. Hill functions which are constructed on the basis of Legendre polynomials have been utilized to solve axisymmetric circular plate problems. The method of Lagrange multipliers is introduced to deal with constraint conditions.

The complexity in the application of hill functions to polar coordinate problems such as circular plates is due to introducing polar coordinate functions into various integrals such as Eqs. (18a, d) and thus usual numerical integration schemes are required to obtain these quantities.

In regard to system equations, Eq. (12a) has its generality but Eq. (12b) is particularly suitable for one-dimensional problems since matrix operations can take advantage of the band-width property. In the treatment of constraint conditions, the method of artificial parameters employed in [6] requires a series of adjustments on the magnitude

TABLE 3

Comparison of the present results and the exact solutions (Ref. [7], shown in parentheses) for a simply supported circular plate with a central hole $(b / a=1 / 10)$, uniformly loaded along inner edge (Fig. 6) (the hill function of order $n=6$ and mesh spacings $m=10$ are used).

\begin{tabular}{|c|c|c|c|c|c|}
\hline$\frac{r}{a}=0.1$ & $\frac{r}{a}=0.2$ & $\frac{r}{a}=0.4$ & $\frac{r}{a}=0.6$ & $\frac{r}{a}=0.8$ & $\frac{r}{a}=1$ \\
\hline \multicolumn{6}{|c|}{ (a) Deflection $y E t^{3} / P a^{2}$. } \\
\hline$(0.632384$ & 0.571552 & 0.442418 & 0.298681 & 0.148493 & 0 \\
\hline 0.631973 & 0.571246 & 0.442228 & 0.298567 & 0.148438 & 0 \\
\hline \multicolumn{6}{|c|}{ (b) Stress along radial direction $-\sigma_{r} t^{2} / P$. } \\
\hline 0 & 0.652505 & 0.492952 & 0.291406 & 0.130385 & 0 \\
\hline 0 & 0.654801 & 0.492650 & 0.291278 & 0.130139 & 0 \\
\hline \multicolumn{6}{|c|}{ (c) Stress along circumferential direction $-\sigma_{\theta} t^{2} / P$. } \\
\hline (3.221540 & 1.708558 & 1.007634 & 0.705834 & 0.509724 & $0.363098)$ \\
\hline 3.222170 & 1.707913 & 1.007047 & 0.705528 & 0.509471 & 0.362928 \\
\hline
\end{tabular}


of parameters, and thus the Lagrange multiplier method appears, at least for the cases studied here, to be more efficient.

From results obtained herein, it seems that the hill function approach does indeed provide very good accuracy and consequently deserves further research for application to various practical problems.

\section{REFERENCES}

[1] O. C. Zienkiewicz, The finite element method in engineering science, McGraw-Hill, London, 1971

[2] J. T. Oden, Finite elements of nonlinear continua, McGraw-Hill, New York, 1972

[3] J. Segethová, Numerical construction of the hill functions, SIAM J. Numer. Anal. 9, 199-204, (1972)

[4] I. Babuska, The finite element method for elliptic differential equations, Lecture at symposium on the numerical solution of partial differential equations, May 1970, University of Maryland

[5] M. H. Schultz, Finite element analysis, Department of Computer Science, Yale University, 1971

[6] R. Kao, Approximate solutions by utilizing hill functions, Computers and Structures 3, 397-412 (1973)

[7] S. Timoshenko and S. Woinowsky-Krieger, Theory of plates and shells, McGraw-Hill, New York, (1959)

[8] H. Goldstein, Classical mechanics, Addison-Wesley Publishing Co., (1965)

[9] T. H. Lee, G. E. Adams, and W. M. Gaines, Computer process control: modeling and optimization, John Wiley and Sons, New York, (1968) 